\title{
On Universality Of Diagenetic Trends
}

\author{
Jack Dvorkin \\ Geophysics Department, Stanford University, Stanford, CA 94305-2215, USA
}

\begin{abstract}
By analyzing rock physics trends from wells located in different parts of the world, one may find a striking similarity among them. This similarity confirms the self-evident truth that there are only a few natural forces -- gravity, water and air transport, temperature, and pressure -- that shape rock, and that these natural forces are the same everywhere on earth.
\end{abstract}

\section{Introduction}

For decades, the main use of seismic data has been to delineate sedimentary bodies and tectonic features in the subsurface. The mission of seeing inside the geological body has been added recently. Mapping porosity, lithology, and other reservoir bulk properties inside the geological body has become possible due to the recent dramatic improvement in seismic acquisition, imaging, and inversion quality as well as the accompanying progress of rock physics.

Rock physics provides transforms between a reservoir's elastic properties and its bulk properties, including porosity, lithology, pore fluid, and pore pressure. Such transforms are known as trends. Trends are built from controlled experiments where the elastic and bulk properties are measured on the same samples at the same conditions. For example, an experimental impedance-porosity trend can be applied to an acoustic impedance volume to map porosity.

The application of a rock physics trend to a volume of seismic data is appropriate if the two are stratigraphically consistent, i.e., the trend represents the depositional and diagenetic variations specific to the volume under examination. Examples are velocity-porosity trends that correspond to changing grain size and sorting due to deposition, or changing amounts of quartz or reactive clay cement due to diagenesis (Dvorkin and Nur, 1996). This is why good-quality modern well log data, where distinctive stratigraphic subsets can be identified, constitute the best basis for building rock physics trends (Gutierrez et al., 2001).

Honoring stratigraphic constraints guarantees that a rock physics trend is deposition- and site-specific. Rationalization by effective-medium modeling makes a trend general, determines the domains of its applicability, and thus reduces the risk of using the trend outside of the initial data range. As an example of rationalization, consider work by Avseth et al. (2000) where empirical data trends are supported by effective-medium curves that represent varying sorting and cementation. Such melding of data and theory is a signature of modern rock physics whose goal is not only to observe and relate but also to explain and generalize.

How general are rational rock physics trends? Can they be applied across tiers of 
deposition and across geographical areas? These are the questions addressed below.

\section{North Sea and Gulf Coast}

Consider first a vertical North Sea well whose log curves of gamma-ray (GR), porosity, and the compressional $(M)$ and shear $(G)$ moduli are shown in Figure 1. The two elastic moduli are obtained from the $P$ - and $S$-wave velocity and bulk density ? as $M ? ? V_{P}^{2}$ and $G ?$ ? $V_{S}^{2}$, respectively, and then theoretically re-calculated for $100 \%$ water saturation via Gassmann's (1951) fluid substitution.

The pay zone is the flat-GR interval at about $1.8 \mathrm{~km}$ depth. It is marked NSB. The shale interval above the pay zone is marked NSA. Both elastic moduli ( $M$ and $G$ ) in the pay zone are much larger than those in the surrounding rock. The observed relatively high stiffness of the pay zone is due to the small amounts of amorphous quartz that cements the grains at their contacts (Figure 2). Such cementation acts to strongly increase the velocity while keeping porosity high (Avseth, 2000).

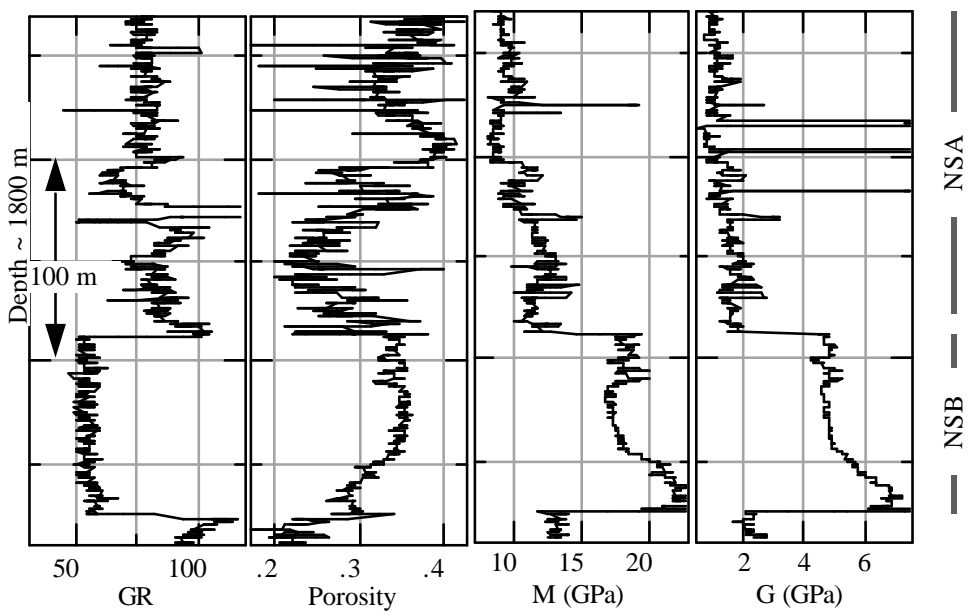

Figure 1. Well log curves for the North Sea well. The elastic moduli are re-calculated for full water saturation from the original data. Courtesy Per Avseth and Norsk Hydro.

The strong elastic contrast between NSA and NSB translates into two distinctive modulus-porosity trends in Figure 2. The lower branches in those plots are called the "uncemented" or friable trends while the upper branches are the "contact cement" trends. 


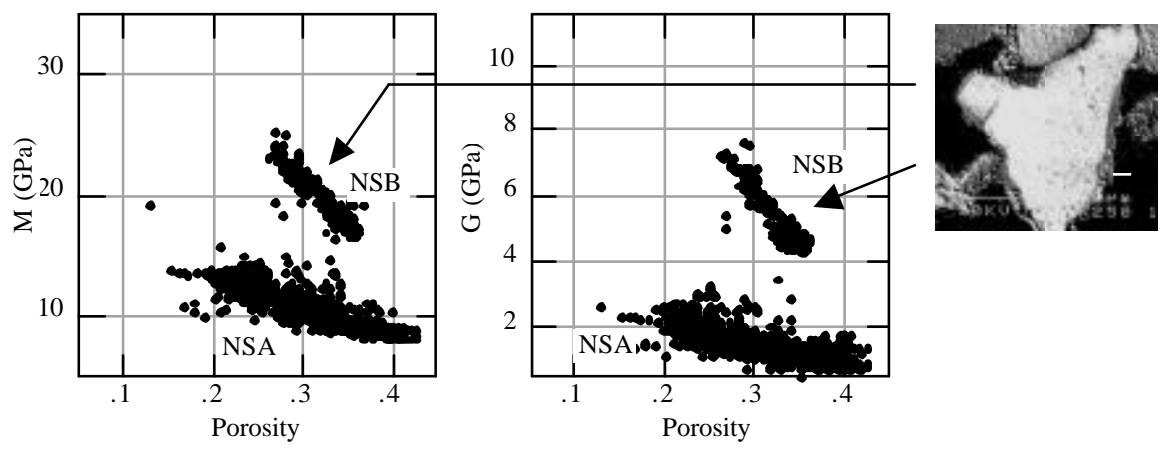

Figure 2. Cross plots based on well log data for the North Sea well. The elastic moduli are re-calculated for full water saturation from the original data. The trends are marked according to the sub-intervals shown in Figure 1, right. The thin section shows contact quartz cement enveloping a quartz grain (courtesy Per Avseth and Norsk Hydro).

Consider next a well located on the US Gulf Coast, several thousand miles away from the North Sea well (log curves are shown in Figure 3). As in the North Sea well, the elastic moduli are theoretically re-calculated for $100 \%$ water saturation. The pay zone is the low-GR interval marked GCB, at a depth of about $3500 \mathrm{~m}$, some $1700 \mathrm{~m}$ deeper than NSB. The shale zone above GCB is marked GCA. The elastic modulus versus porosity cross-plots for the Gulf Coast well (Figure 4), similar to those for the North Sea well, exhibit clear separation between the pay zone and the shaley remainder of the well.

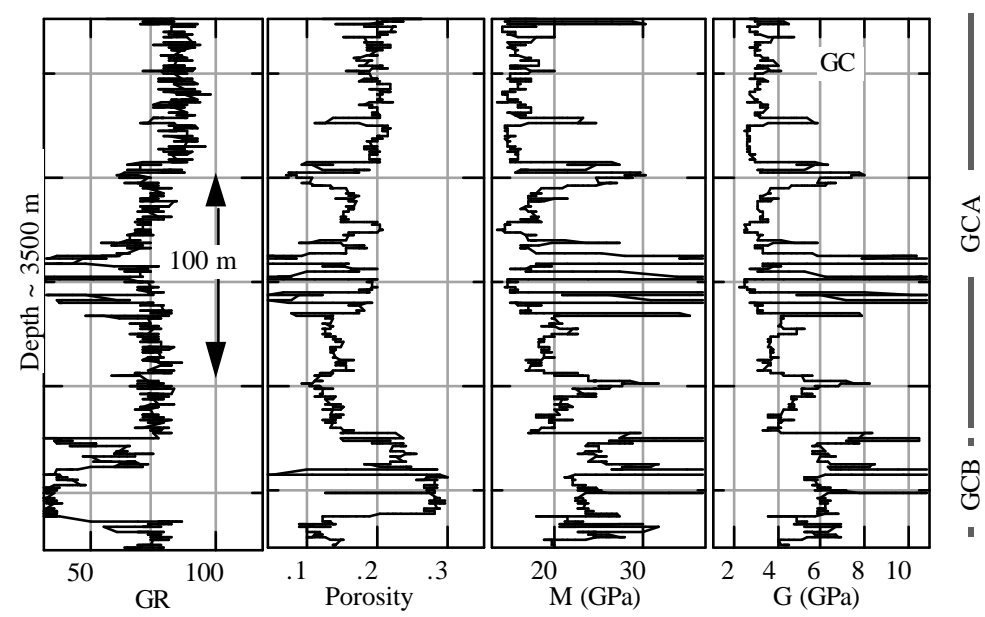

Figure 3. Well log curves for the Gulf Coast well. The elastic moduli are re-calculated for full water saturation from the original data. 

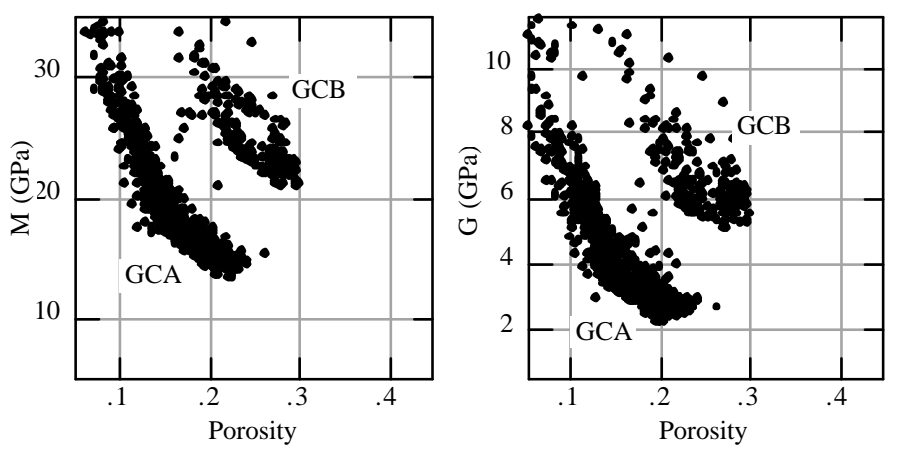

Figure 4. Cross plots based on well log data for the Gulf Coast well. The elastic moduli are re-calculated for full water saturation from the original data. The trends are marked according to the sub-intervals shown in Figure 3, right.

Consider now superposition of cross-plots for the two wells (Figure 5). In spite of the large geographical and depth difference between the wells, the elastic moduli versus porosity trends for the Gulf Coast well seamlessly continue the trends for the North Sea well. The trends are supported by the contact cement effective-medium model (the pay zones) and the uncemented-sand (shaley intervals). The burial-related compaction and cementation processes in the Gulf Coast well continue those started in the North Sea well.

\section{Conclusion}

The apparent universality of compaction and diagenesis trends in the rock physics space, as observed in two earth intervals separated by vast geographical distance and located ad different depths, indicates that rock physics transforms valid at an existing well may be applicable to deriving reservoir properties away from this well control, based on seismic data.
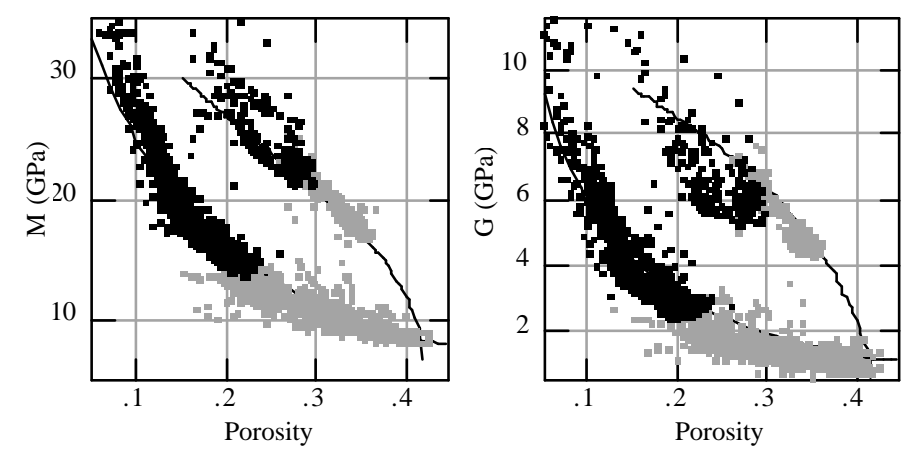

Figure 5. Modulus versus porosity cross plots for the North Sea (gray) and Gulf Coast (black) wells. The curves are from the theoretical contact cement model (upper branch) and uncemented model (lower branch). 


\section{References}

Avseth, P., Dvorkin, J., Mavko, G., and Rykkje, J., 2000, Rock physics diagnostic of North Sea sands: Link between microstructure and seismic properties, Geophysical Research Letters, 27, 2761-2764.

Dvorkin, J., and Nur, A., 1996, Elasticity of High-Porosity Sandstones: Theory for Two North Sea Datasets, Geophysics, 61, 1363-1370.

Gassmann, F., 1951, Elasticity of porous media--Uber die elastizitat poroser medien, Vierteljahrsschrift der Naturforschenden Gesselschaft, 96, 1-23.

Gutierrez, M.A., Dvorkin, J., and Nur, A., 2001, Stratigraphy-guided rock physics, The Leading Edge, 21, \#1, 98-103. 